\title{
CONTABILIDADE DE GESTÃO DA SUSTENTABILIDADE: REVISÃO SISTEMÁTICA DA LITERATURA MUNDIAL ${ }^{1}$
}

\author{
SUSTAINABILITY MANAGEMENT ACCOUNTING: SYSTEMATIC REVIEW OF \\ WORLD LITERATURE
}

\section{CONTABILIDAD DE GESTIÓN DE LA SOSTENIBILIDAD: REVISIÓN SISTEMÁTICA DE LA LITERATURA MUNDIAL}
Daniele Rodrigues Garcia, Mestranda em Administração pelo Programa de Pós Graduação em Administração da Universidade Federal do Rio Grande (PPGA/FURG).
Formação: Tecnóloga em Administração de Pequenas e Médias Empresas pela Universidade
Norte do Paraná (UNOPAR). email: furg.danielegarcia@gmail.com
Orcid: http://orcid.org/0000-0002-5016-3284
Currículo Lattes: http://lattes.cnpq.br/2580415679239791

Errol Fernando Zepka Pereira Junior, Mestrando em Administração pelo Programa de Pós Graduação em Administração da Universidade Federal do Rio Grande (PPGA/FURG).

email: zepkaef@gmail.com

ORCID: http://orcid.org/0000-0002-4203-0801

Currículo Lattes: http://lattes.cnpq.br/2859488522345809

\begin{abstract}
RESUMO
A sustentabilidade tem sido alvo de inúmeros debates e estudos há décadas, no meio acadêmico, empresarial e governamental. Do ponto de vista comum, seu conceito está relacionado ao desenvolvimento sustentável universal, ou seja, é formada por atitudes ambientalmente corretas, economicamente viáveis e socialmente justas. Já a contabilidade de gestão da sustentabilidade é um termo que geralmente é utilizado para avaliar o alinhamento interno em relação às questões sustentáveis e melhorar o desempenho das organizações como um todo. Estudos sobre este tema apresentam amplitude, porém carecem de investigação focada para identificar, selecionar, avaliar e sintetizar as evidências relevantes disponíveis, oferecendo um panorama que sirva de base aos estudos futuros, bem como identificando lacunas que possam ser preenchidas por meio de novos estudos, no que se justifica a proposta do presente estudo. O objetivo geral deste estudo é levantar o panorama da produção acadêmica nos principais periódicos científicos sobre este tema através de uma revisão sistemática da literatura. $\mathrm{O}$ principal achado da pesquisa concentra-se em como a contabilidade de gestão da sustentabilidade tem sido estudada na literatura e quais as principais relações detectadas, sendo elas: contadores, controle e medição, gestão e desempenho.
\end{abstract}

Palavras-chave: Contabilidade. Gestão. Custos. Desempenho. Sustentabilidade.

1 Artigo submetido em 22/01/2019, revisado em 25/07/2019, aceito em 26/07/2019 e divulgado em 20/10/2019 pelo EditorAlexandre Rabêlo Neto, após double blind review.

GєCont, v.6, n. 1, Floriano-PI, Jan-Jun. 2019. 


\section{ABSTRACT}

Sustainability has been the subject of countless debates and studies for decades in academia, business and government. From a common point of view, its concept is related to universal sustainable development, that is, it is formed by environmentally correct, economically viable and socially just attitudes. Sustainability management accounting, on the other hand, is a term that is generally used to assess internal alignment with respect to sustainable issues and to improve the performance of organizations as a whole. Studies on this topic are broad, but lack focused research to identify, select, evaluate and synthesize the relevant evidence available, providing a framework for future studies, as well as identifying gaps that can be filled through further studies, justifying the proposal of the present study. The general objective of this study is to raise the panorama of academic production in the main scientific journals on this topic through a systematic review of the literature. The main finding of the research focuses on how sustainability management accounting has been studied in the literature and what the main relationships detected are: counters, control and measurement, management and performance.

Keywords: Accounting. Management. Costs. Performance. Sustainability.

\section{RESUMEN}

La sostenibilidad ha sido objeto de numerosos debates y estudios desde hace décadas, en el medio académico, empresarial y gubernamental. Desde el punto de vista común, su concepto está relacionado al desarrollo sostenible universal, es decir, la misma está formada por actitudes ambientalmente correctas, económicamente viables y socialmente justas. La contabilidad de gestión de la sostenibilidad es un término que generalmente se utiliza para evaluar la alineación interna en relación con las cuestiones sostenibles y mejorar el desempeño de las organizaciones en su conjunto. El objetivo general de este estudio es levantar el panorama de la producción académica en los principales periódicos científicos sobre este tema a través de una revisión sistemática de la literatura. El principal hallazgo de la investigación se concentra en cómo la contabilidad de gestión de la sostenibilidad ha sido estudiada en la literatura y cuáles las principales relaciones detectadas, siendo ellas: contadores, control y medición, gestión y desempeño.

Palabras clave: Contabilidad. Gestión. Costas. Rendimiento. Sostenibilidad.

\section{INTRODUÇÃO}

A sustentabilidade é um conceito relacionado ao desenvolvimento sustentável, formado por um conjunto de ideias, estratégias e atitudes ecologicamente corretas, economicamente viáveis e socialmente justas com o objetivo de garantir a sobrevivência dos recursos naturais do planeta para esta e as próximas gerações (LUNARDI; FRIO; BRUM, 2011). Este termo incorporou-se muito rápido aos vocabulários politicamente corretos da sociedade, das organizações e do governo, contudo, apesar dos inúmeros discursos voltados para a necessidade urgente de ação, os esforços efetivos neste sentido parecem não apresentar os resultados necessários, tornando imprescindível que o principal foco dos estudos acadêmicos volte-se para facilitar no entendimento e no combate às causas da insustentabilidade, na tentativa de gerar dados e informações que apresentem alternativas e soluções para o mundo.

Da Silva et al. (2019) explicam que a pressão pelo desenvolvimento de práticas produtivas mais sustentáveis não vem sendo feita apenas pelos governos, mas também pela sociedade, que tem cada vez mais cobrado medidas das empresas para melhorar a gestão dos resíduos sólidos gerados pelos processos produtivos. Os autores entendem ainda que as 
empresas que investem de forma estratégica em formas produtivas e logísticas mais sustentáveis ganham competição de mercado.

A contabilidade de gestão da sustentabilidade (CGS) surge neste cenário, portanto, para auxiliar nesta tarefa, servindo de ferramenta para gerenciar a sustentabilidade das organizações e esta pesquisa se justifica, pois, a CGS usa não apenas dados monetários, mas também dados não monetários (físicos) que frequentemente reflete os direcionadores de resultados monetários (BURRITT et al., 2002; SCHALTEGGER; BURRITT, 2000). O interesse em informação não monetária aumentou nos últimos anos com o crescente reconhecimento de que as questões ambientais e sociais, reforçando que coletar dados sobre desempenho econômico, social e ambiental ajuda as empresas a entender o valor que elas criam (IIRC, 2011) e pode influenciar positivamente a tomada de decisões gerenciais (SÁNCHEZ et al., 2013).

Ainda que exista uma necessidade e grande prospecção de pesquisas no tema da sustentabilidade, se encontra na literatura nacional e internacional alguns estudos mostrando a restrição de pesquisa sobre formas de gestão ou mesmo produções mais sustentáveis. O estudo de Martens, Brones e Carvalho (2013) procurou apresentar as lacunas e tendências de pesquisa na literatura de sustentabilidade e gerenciamento. Para isso, os autores trabalharam em uma revisão sistemática de literatura e análise de conteúdo para identificação e interpretação das principais publicações acerca do tema na literatura científica em um período de vinte anos. Foram encontrados a quantidade restrita de apenas vinte e três artigos. Já o trabalho de Pereira et al. (2018) caracterizou as publicações na temática de produções enxutas e sustentáveis através de uma análise bibliométrica. Nesta pesquisa, em um espectro temporal de 10 anos (entre 2007 e 2017), os autores ressaltaram a quantidade restrita de artigos encontrados, apenas 19 artigos em três renomadas bases de dados internacionais, a saber: Science Direct, Scopus e Web of Science.

Diante do exposto, percebe-se que os estudos sobre o tema apresentam amplitude, os quais carecem de investigação focada para identificar, selecionar, avaliar e sintetizar as evidências relevantes disponíveis, oferecendo um panorama que sirva de base aos estudos futuros, bem como identificando lacunas que possam ser preenchidas por meio de novos estudos. Neste sentido, o presente estudo visa contribuir com um maior entendimento sobre a contabilidade de gestão de sustentabilidade, bem como, vislumbrar oportunidades de contribuição sobre essa perspectiva. Assim, o presente artigo busca investigar o seguinte problema de pesquisa: Quais os principais avanços da Contabilidade Gerencial em relação a Gestão da Sustentabilidade?

Para responder à pergunta do problema de pesquisa, o objetivo geral deste estudo é levantar o panorama da produção acadêmica nos principais periódicos científicos sobre este tema e, a partir do mesmo, definem-se os seguintes objetivos específicos: (i) levantar como a contabilidade de gestão da sustentabilidade tem sido estudada na literatura e quais as principais relações detectadas; (ii) levantar os principais procedimentos metodológicos utilizados na literatura; (iii) levantar os termos mais recorrentes utilizados na literatura.

Uma revisão sistemática foi conduzida para explorar como a contabilidade de gestão da sustentabilidade tem sido pesquisada na literatura ao longo do tempo. Para tanto, adotou-se um processo científico rigoroso de busca e avaliação da literatura segundo Tranfield et al. (2003). Neste estudo, a revisão sistemática seguiu os protocolos delineados por Sampaio e Mancini (2007), Petticrew e Roberts (2006) e Tranfield et al. (2003).

Este artigo constitui-se de cinco seções: na primeira apresenta-se a introdução; na segunda o referencial teórico, abordando a sustentabilidade, a gestão e a contabilidade gerencial e, por fim, a gestão da contabilidade da sustentabilidade; na terceira, o método; na quarta, os resultados e discussão; e, por fim, as considerações finais. 


\section{REFERENCIAL TEÓRICO}

\subsection{SUSTENTABILIDADE}

Há algumas décadas o tema sustentabilidade vem sendo alvo de inúmeros debates e estudos no meio acadêmico, empresarial e governamental. Uma das definições de sustentabilidade mais difundida é a da Comissão Brundtland (WCED, 1987), a qual considera que o desenvolvimento sustentável deve satisfazer às necessidades da atual geração sem comprometer as necessidades das gerações futuras. A verdade é que, desde esta definição, já surgiram inúmeras ao longo do tempo e, com certeza, existirão muitas outras, contudo, o ponto comum em todas elas, quando analisadas detalhadamente, está nas dimensões que compõem o termo sustentabilidade.

A maioria dos estudos afirma que sustentabilidade é composta de três dimensões interrelacionadas: econômica, ambiental e social. Essas dimensões são também conhecidas como tripple bottom line. Assim sendo, Almeida (2002) reforça que a dimensão econômica inclui não só a economia formal, mas também as atividades informais que provêm serviços para os indivíduos e grupos e aumentam, assim, a renda monetária e o padrão de vida dos indivíduos; a dimensão ambiental ou ecológica estimula empresas a considerarem o impacto de suas atividades sobre o meio ambiente, na forma de utilização dos recursos naturais, e contribui para a integração da administração ambiental na rotina de trabalho, e; a dimensão social consiste no aspecto social relacionado às qualidades dos seres humanos, como suas habilidades, dedicação e experiências, abrangendo tanto o ambiente interno da empresa quanto o externo.

O tema ganhou em 2005, através de uma iniciativa pioneira na América Latina, um índice que busca criar um ambiente de investimento compatível com as demandas de desenvolvimento sustentável da sociedade contemporânea e estimular a responsabilidade ética das corporações denominado Índice de Sustentabilidade Empresarial (ISE). O ISE é uma ferramenta para análise comparativa da performance das empresas listadas na B3 sob o aspecto da sustentabilidade corporativa, baseada em eficiência econômica, equilíbrio ambiental, justiça social e governança corporativa. Também amplia o entendimento sobre empresas e grupos comprometidos com a sustentabilidade, diferenciando-os em termos de qualidade, nível de compromisso com o desenvolvimento sustentável, equidade, transparência e prestação de contas, natureza do produto, além do desempenho empresarial nas dimensões econômicofinanceira, social, ambiental e mudança do clima.

\subsection{GESTÃO E CONTABILIDADE GERENCIAL}

Segundo Barbieri (2007), o grande desafio para as empresas é a adaptação de sua gestão às demandas do meio ambiente. As preocupações ambientais não surgem espontaneamente, são influenciadas por três grandes conjuntos de forças que interagem reciprocamente: governo, sociedade e mercado. Junta-se a isto, a necessidade de satisfazer a exigência por uma melhor qualidade de vida e o subsequente consumo de produtos e serviços de maneira que não seja cumulativamente destrutivo para os recursos e ameaçador para a vida numa escala planetária (DIAS; MOURA, 2007).

Höjer et al. (2008) reconhecem que a integração dos aspectos ambientais no planejamento das organizações exige o desenvolvimento de ferramentas poderosas e eficientes para tornar possível a compreensão e avaliação dos sistemas econômicos, tecnológicos e ambientais, e também para possibilitar a essas organizações a formação de uma estrutura eficiente de informações, necessária à alimentação dos seus processos de tomada de decisões.

Gerenciar a sustentabilidade de uma organização é fornecer conhecimento sobre os impactos sociais e ambientais desejados e indesejados, ideias sobre como estes impactos podem 
ser minimizados, e para obter informações sobre se as mudanças implementadas realmente criam as contribuições desejadas para o desenvolvimento sustentável (EPSTEIN; BUHOVAC, 2014). No entanto, um pré-requisito crucial para a gestão da sustentabilidade é desenvolver capacidades para medir e monitorar esses valores novos ou adicionais (MAAS et al., 2016).

\subsection{CONTABILIDADE DE GESTÃO DA SUSTENTÁVEL}

Segundo Burritt (2010), ao referir-se ao processo de coleta, análise e comunicação de informações relacionadas com a sustentabilidade existe um termo denominado "contabilidade de gestão de sustentabilidade" que em geral é utilizado para avaliar o alinhamento interno e melhorar o desempenho.

Para Laurinkeviciute e Stasiskiene (2001) a contabilidade de gestão de sustentabilidade, busca integrar a avaliação de sustentabilidade à contabilidade gerencial, ao controle da gestão e aos relatórios, baseando-se na análise financeira, orientada para objetivos estratégicos de sustentabilidade para facilitar o processo de tomada de decisão pelos gestores superiores.

As empresas optam por implantar a contabilidade de gestão da sustentabilidade para satisfazer as partes interessadas, conforme descobertas de Gunarathne et al. (2016) e, conforme os fatores externos e fatores internos às empresas. Os fatores externos vão desde a sua sobrevivência (LAURINKEVICIUTE; STASISKIENE, 2001); suprir expectativas e pressões das partes interessada, as quais são dependentes de instituições nacionais, históricas, culturais, sociais (GUNARATHNE et al., 2016) e órgão regulamentares (MAAS et al., 2016), criando desta forma valor corporativo (GUNARATHNE et al., 2016), até diminuir impactos ambientais e sociais. Já os fatores internos passam por medir e avaliar o desempenho ambiental, social e econômico (Lee e Wu, 2014), os riscos (JASCH; LAVICKA, 2006), os custos (EGAN; TWEEDIE, 2018; LEE; WU, 2014; JASCH; LAVICKA, 2006), a saúde e a segurança (JASCH; LAVICKA, 2006), a produção mais limpa (LEE, 2012), a logística (LEE; WU, 2014), a cadeia de suprimentos (LEE, 2012; LEE; WU, 2014), a emissão de carbono (LEE, 2012; LEE; WU, 2014); orientar para decisões corporativas (LEE, 2012) e mudanças organizacionais (GUNARATHNE et al., 2016; SCHALTEGGER; ZVEZDOV, 2015), implantar ou ampliar as práticas de contabilidade gerencial (SCHALTEGGER; ZVEZDOV, 2015; SILVA; GUENTHER, 2018), gerenciamento de informações e verdes ou de sustentabilidade (SCHALTEGGER; ZVEZDOV, 2015); medir o desempenho (JASCH; LAVICKA, 2006; LEE; WU, 2014; SCHALTEGGER; ZVEZDOV, 2015; SILVA; GUENTHER, 2018); controlar a gestão como um todo (MAAS et al., 2016); e, ainda, avaliar pré e pós investimento (JASCH; LAVICKA, 2006).

Podem operacionalizar a contabilidade de gestão da sustentabilidade contadores de formação, os quais aparecem parcialmente envolvidos na gestão (EGAN; TWEEDIE, 2018), apenas como "guardiões da informação sobre sustentabilidade" e ocupando uma posição de manutenção entre os gestores de sustentabilidade e os gestores superiores em que se defende cada mais suas participações devido a duas questões primordiais: (i) melhorar a educação de sustentabilidade; (ii) melhorar os incentivos e estruturas que motivam a contabilização (SCHALTEGGER; ZVEZDOV, 2015).

Diversos são os sistemas e ferramentas/recursos disponíveis para a execução da contabilidade de gestão da sustentabilidade e, por conseguinte, para facilitar a tomada de decisão (LAURINKEVICIUTE; STASISKIENE, 2001) pelos gestores, os quais, necessitam apenas de adaptações para atender ao tripé ambiental, social e econômico e, desta maneira, formar o "Sistema Integrado de Gestão Sustentável" (LAURINKEVICIUTE; STASISKIENE, 2001; MAAS et al., 2016) com o objetivo de poupar esforços de administração, aumentar o controle gerencial, otimizar o volume de informações e maximizar resultados. Entre as ferramentas existentes e frequentemente utilizadas encontra-se: Sistema de Controle de Gestão 
- Eco-controle (LEE, 2012); Sistema de Avaliação de Desempenho; Sistema de Controle de Custos; Sistema de Gestão de Risco; Sistema de Controle de Segurança (GUNARATHNE et al., 2016); Sistema de Gestão de Emissão de Carbono (LEE, 2012), etc. E, como ferramentas/recursos, os estudos apontam os indicadores de desempenho (JASCH; LAVICKA, 2006; GUNARATHNE et al., 2016; LEE; WU, 2014); os relatórios (MAAS et al., 2016); e, as consultorias externas (JASCH; LAVICKA, 2006).

\section{METODOLOGIA}

Os procedimentos metodológicos deste artigo apresentam-se baseados na estrutura proposta por Roesch (2010), e são escritos quanto ao seu propósito, seu caráter, sua abordagem e o delineamento das técnicas de coleta e técnicas de análise dos dados.

Quanto ao propósito, a pesquisa está classificada como pesquisa diagnóstico. Para Roesch (2010), a pesquisa diagnóstico pretende explorar o ambiente organizacional e de mercado, levantar e definir problemas. Nesse trabalho, a pesquisa objetivou explorar a produção científica referente a contabilidade de gestão da sustentabilidade.

A respeito do caráter do estudo, este se enquadra como uma pesquisa exploratória, cujo objetivo principal é a identificação e análise qualitativa da produção científica no que tange à contabilidade de gestão da sustentabilidade.

Quanto à abordagem, este artigo pode ser classificado como qualitativo. Para Neves (1996) a pesquisa qualitativa pode ser definida como um conjunto de diferentes técnicas interpretativas onde procura-se descrever e decodificar os componentes onde há um sistema complexo com muitos significados, tendo por objetivo traduzir e expressar o sentido dos fenômenos do mundo social para o pesquisado

Quanto à técnica de coleta de dados, a pesquisa é bibliográfica, caracterizando-se como a fonte de dados secundários deste estudo. Segundo Gil (2010), esta pesquisa é elabora-se com base em materiais já publicados, tais como: livros, revistas, jornais, teses, dissertações e anais de eventos científicos. Neste trabalho, mais precisamente apresenta-se uma pesquisa bibliográfica através da revisão sistemática de literatura acerca da contabilidade de gestão da sustentabilidade

Uma revisão sistemática foi conduzida para explorar como a "contabilidade de gestão da sustentabilidade" tem sido pesquisada na literatura. Este estudo se concentra em trabalhos de pesquisa que demostrem as mais variadas formas de utilização e/ou recomendação da contabilidade de gestão da sustentabilidade no mundo, nos mais diversos tipos e tamanhos de empresas, iniciativas e práticas internas e externas, profissionais envolvidos, fenômenos observados, impactos causados, perspectivas futuras, agendas globais, apesar das escassas publicações sobre o tema. A revisão sistemática da literatura contribui para responder a perguntas sobre o que funciona e o que não funciona ponto de vista prático (Petticrew e Roberts, 2006).

Uma revisão sistemática deve adotar um processo científico e rigoroso de busca e avaliação da literatura de uma maneira que o processo de busca possa ser facilmente entendido e replicado (TRANFIELD et al., 2003). Neste estudo, a revisão sistemática seguiu os protocolos delineados por Sampaio e Mancini (2007), Petticrew e Roberts (2006) e Tranfield et al. (2003). A figura 1 mostra o desenho do protocolo de pesquisa. Neste esquema, as células do lado direito representam critérios de inclusão e artigos incluídos nas fases dadas, enquanto as células o lado esquerdo representa documentos que não correspondem aos critérios de inclusão e tiveram que ser retirados da revisão sistemática.

Posteriormente, realizou-se a análise dos conceitos apresentados, através de análise de conteúdo categorial - um método frequentemente utilizado quando se refere à análise de dados qualitativos. Segundo Bardin (1977, p. 119), “a categorização é uma operação de classificação 
de elementos constitutivos de um conjunto, por diferenciação e, seguidamente, por reagrupamentos segundo o gênero (analogia) com os critérios previamente definidos". As categorias possuem um título genérico e são compostas por um grupo de elementos agrupados em razão de suas similaridades. O pesquisador é o responsável por delimitar as unidades de codificação, que podem ser uma palavra ou uma frase.

O procedimento e o resultado da etapa inicial da revisão sistemática podem ser encontrados na figura 1 - o desenho do protocolo de pesquisa.

\section{Figura 1: Desenho do protocolo de pesquisa}

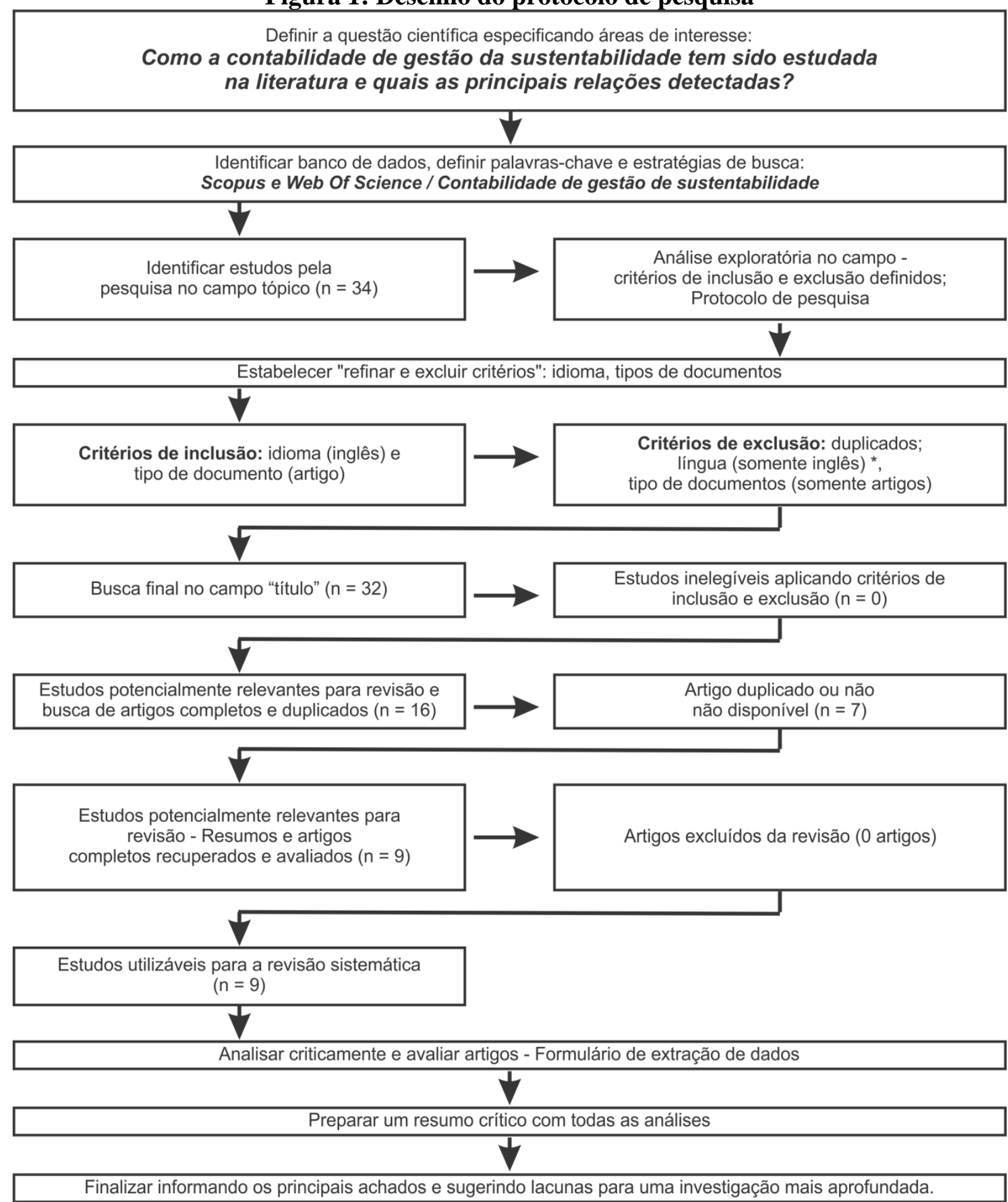

Fonte: dados da pesquisa. 


\section{RESULTADOS E DISCUSSÃO}

Os resultados indicam que ainda existem poucos estudos sobre o termo "contabilidade de gestão da sustentabilidade", o qual foi pesquisado nas bases Scopus e Web Of Science como "Sustainability management accounting" e, desde 2006 apenas 9 artigos foram publicados, conforme pode ser percebido na figura 2.

Figura 2: Distribuição dos artigos incluídos na revisão sistemática por ano.

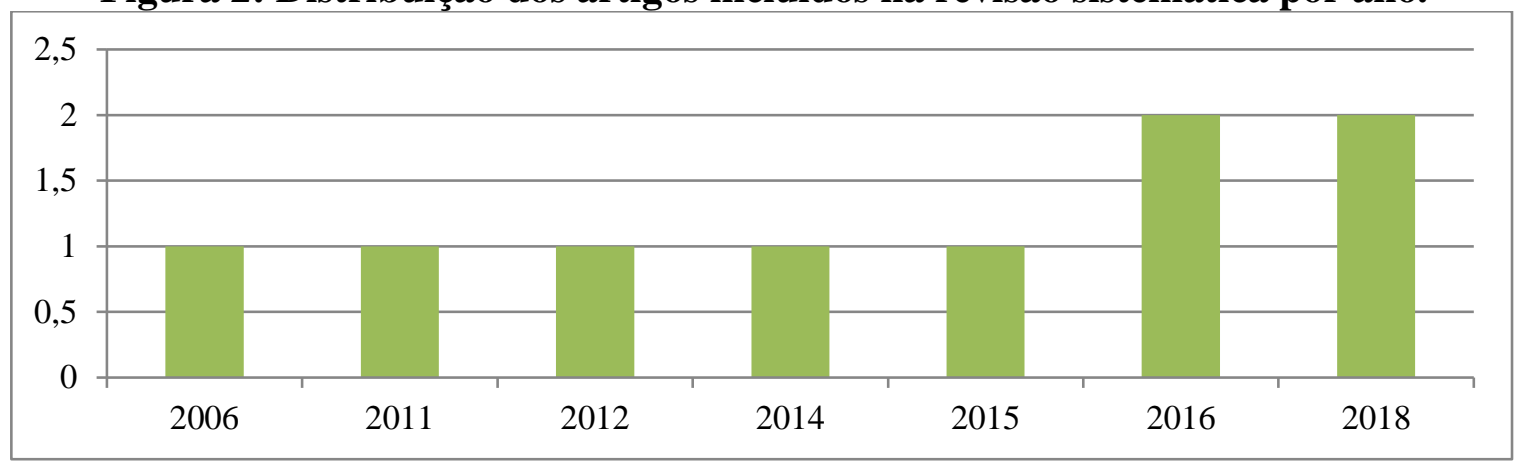

Fonte: dados da pesquisa

\subsection{COMO A CONTABILIDADE DE GESTÃO DA SUSTENTABILIDADE TEM} SIDO ESTUDADA NA LITERATURA E QUAIS AS PRINCIPAIS RELAÇÕES DETECTADAS

Investigando a literatura sobre a contabilidade de gestão da sustentabilidade foram detectadas quatros relações principais: contadores, controle e medição, desempenho, e, gestão, conforme consta na Tabela 1.

Tabela 1: Como a contabilidade de gestão da sustentabilidade tem sido estudada e quais as principais relações detectadas

\begin{tabular}{|c|c|c|c|}
\hline $\begin{array}{l}\text { Abordagens } \\
\text { principais }\end{array}$ & & Definição & Referências \\
\hline Contadores & & $\begin{array}{l}\text { A contribuição dos contadores nas iniciativas de } \\
\text { sustentabilidade organizacional e o envolvimento dos } \\
\text { contadores na prática corporativa de gestão de informações } \\
\text { de sustentabilidade }\end{array}$ & $\begin{array}{l}\text { Egan e Tweedie (2018); } \\
\text { Schaltegger e Zvezdov } \\
(2015)\end{array}$ \\
\hline $\begin{array}{l}\text { Controle } \\
\text { medição }\end{array}$ & $\mathrm{e}$ & $\begin{array}{l}\text { Os papéis e a utilidade do eco-controle como um meio de } \\
\text { identificar e medir o desempenho de carbono; A utilidade dos } \\
\text { controles de segurança e da contabilidade na gestão da } \\
\text { sustentabilidade social corporativa; Medição e gestão de } \\
\text { questões de sustentabilidade; Integração de indicadores de } \\
\text { desempenho de diferentes perspectivas e modelo de medição } \\
\text { do sistema para melhoria do desempenho de sustentabilidade; } \\
\text { Mensuração do desempenho de sustentabilidade }\end{array}$ & $\begin{array}{l}\text { Lee (2012); Gunarathne et al. } \\
\text { (2016); Maas et al. (2016); } \\
\text { Lee e Wu (2014); Silva e } \\
\text { Guenther (2014) }\end{array}$ \\
\hline Desempenho & & $\begin{array}{l}\text { Os papéis e a utilidade do eco-controle como um meio de } \\
\text { identificar e medir o desempenho de carbono; Integração de } \\
\text { indicadores de desempenho de diferentes perspectivas e } \\
\text { modelo de medição do sistema para melhoria do desempenho } \\
\text { de sustentabilidade; A avaliação dos custos de gestão da } \\
\text { sustentabilidade na melhoria do desempenho ambiental, } \\
\text { social e econômico; Mensuração do desempenho de } \\
\text { sustentabilidade; e Melhorar desempenho de sustentabilidade }\end{array}$ & $\begin{array}{l}\text { Lee (2012); Lee e Wu (2014); } \\
\text { Jasch e Lavicka (2006); Silva } \\
\text { e Guenther (2014); } \\
\text { Laurinkeviciute e Stasiskiene } \\
\text { (2011) }\end{array}$ \\
\hline
\end{tabular}




\begin{tabular}{l|l|l}
\hline \multirow{3}{*}{ Gestão } & $\begin{array}{l}\text { Medição e gestão de questões de sustentabilidade e a } \\
\text { avaliação dos custos de gestão da sustentabilidade na } \\
\text { melhoria do desempenho ambiental, social e econômico. }\end{array}$ & $\begin{array}{l}\text { Maas et al. (2016); Jasch e } \\
\text { Lavicka (2006) }\end{array}$ \\
\hline
\end{tabular}

Fonte: dados da pesquisa.

\subsubsection{RELAÇÕES DETECTADAS SOBRE OS CONTADORES}

A abordagem relacionada aos contadores constou em dois artigos com temáticas bem semelhantes, o primeiro deles foi publicado por Schaltegger e Zvezdov (2015) e levantou a questão do envolvimento dos contadores nas práticas corporativas de gestão de informação de sustentabilidade. A análise empírica é baseada em uma estrutura para identificar as diferentes taxas de sustentabilidade e contabilidade. Os caminhos possíveis de desenvolvimento para um envolvimento construtivo mais longo dos contadores são identificados - para melhorar a educação para contadores, uma vez que o conhecimento de sustentabilidade é um grande obstáculo e / ou melhorar os incentivos e estruturas que motivam a contabilização.

O outro artigo, publicado por Egan e Tweedie (2018), explorou como os contadores podem contribuir para iniciativas de sustentabilidade organizacional a partir de um estudo de caso. Como resultado, embora os contadores tenham se adaptado bem às mudanças iniciais alinhadas à eficiência de custos, eles se esforçaram para se engajar em melhorias mais criativas de sustentabilidade. $\mathrm{O}$ artigo explica tanto as adaptações quanto as restrições como interações entre o habitus profissional dos contadores, as capitais e seu campo organizacional mais amplo. Estratégias anteriores para envolver contadores (por exemplo, treinamento) abordam apenas parcialmente esses fatores. A profissão contábil tem insistentemente incitado os membros a contribuir para iniciativas de sustentabilidade organizacional. Este artigo forneceu uma visão de como as organizações podem combinar a aculturação profissional e os capitais apropriados para avançar nessa agenda, embora a ecoeficiência seja apenas um elemento potencial do gerenciamento abrangente da sustentabilidade organizacional, os insights do artigo sobre contabilistas envolventes contribuem para entender como agendas mais amplas de sustentabilidade social podem ser promovidas.

\subsubsection{RELAÇÕES DETECTADAS SOBRE CONTROLE E MEDIÇÃO}

A abordagem sobre controle e medição apresenta-se de forma mais incisiva, complexa e ampla em cinco dos nove artigo pesquisados. No primeiro artigo publicado, de autoria de Lee (2012) abordou-se que a gestão das emissões de carbono e o desenvolvimento de uma produção mais limpa pode ser um desafio exigente para os negócios e a indústria, que a contabilização do gerenciamento de carbono requer quantificação das emissões diretas e indiretas, que há uma falta de conceitos e ferramentas adequadas para uso na medição do desempenho de carbono tanto no processo de produção como na cadeia de suprimentos como um todo. $\mathrm{O}$ artigo teve o propósito de explorar o papel da contabilidade gerencial e o meio ambiente, em particular, sobre o controle ecológico para a gestão do carbono e a gestão da cadeia de suprimentos de uma empresa. $\mathrm{O}$ estudo descobriu que o controle ecológico pode promover o alinhamento entre estratégia de gerenciamento de carbono e medição de desempenho de carbono da empresa, e fornece informações para tomadores de decisões corporativas. Além disso, o mapeamento viável do fluxo de carbono na produção fornece oportunidades importantes para melhorar o desempenho de carbono na cadeia de suprimentos.

No segundo artigo publicado por Lee e Wu (2014), a abordagem refere-se a maneira pela qual o desempenho econômico e ambiental pode ser medido simultaneamente, adotando uma abordagem multi-metodológica para a logística e gestão da cadeia de suprimentos, a fim de enfrentar os desafios da sustentabilidade. A abordagem multi-metodológica depende dos 
méritos das diferentes metodologias, fornece mais flexibilidade na solução de problemas sob investigação e tolera estimativas imprecisas de parâmetros durante o processo. O caso valida a aplicabilidade e utilidade da abordagem e destaca os resultados comparativos dos custos e das emissões na logística de transporte de mercadorias. O transporte ferroviário é identificado como dando a oportunidade estudar a rede de distribuição de contêineres e cargas de curta distância, embora inicialmente, isso não parece ser a opção mais econômica. Este estudo descobriu que é melhor considerar simultaneamente indicadores de desempenho de diferentes perspectivas e integrá-los em um modelo de medição do sistema para que as corporações possam melhorar seu desempenho de sustentabilidade.

No terceiro artigo, publicado no mesmo ano do anterior, de Silva e Guenther (2014) apresentou as áreas potenciais de pesquisa para mensuração desempenho de sustentabilidade que surgiu em um workshop conjunto de pesquisadores e uma Conferência de Gestão de Contexto Ambiental e Sustentabilidade na Europa, organizada em cooperação com o Conselho Empresarial Mundial para o Desenvolvimento Sustentável através da abordagem do World Café Method, onde os principais tópicos discutidos pelos participantes da conferência foram identificados e uma visão geral das áreas potenciais foram estabelecidas pesquisas sobre "medição do desempenho de sustentabilidade", com foco no impacto da mensuração e do valor da sustentabilidade. Os autores acreditam que o diagrama de fluxo de processos generalizado criado a partir do evento pode atuar como ponto de partida para facilitar grandes grupos transdisciplinares.

Já o quarto artigo, de Maas et al. (2016) faz algumas reflexões importantes e identifica que muito tem sido escrito sobre "por que" as empresas estão envolvidas em questões de sustentabilidade, no entanto, relativamente pouca pesquisa aborda a questão integrativa "como", particularmente "Como as empresas podem e integram a avaliação da sustentabilidade, a contabilidade gerencial, controle de gestão e relatórios?"A sustentabilidade corporativa, no entanto, requer medição e gestão de questões de sustentabilidade, em vez de aplicações isoladas de diferentes ferramentas na organização. Este artigo analisa a literatura tratando de links e parcial ligações entre avaliação de sustentabilidade, contabilidade de gestão, controle de gestão e relatórios. Os principais resultados mostram que os vários conceitos (avaliação de desempenho, contabilidade gerencial, controle gerencial e relatórios) são definidos e utilizados em várias maneiras, mas principalmente tratadas de maneira isolada.

E, o último artigo e mais recente artigo, de Gunarathne et al. (2016) tratou da utilidade dos controles de segurança e da contabilidade na gestão da sustentabilidade social corporativa, respondendo às demandas e expectativas dos stakeholders. O estudo (i) identificou uma relação recíproca entre as estratégias de gestão das partes interessadas e o sistema de controle de segurança que inclui uma combinação de principais indicadores de desempenho de segurança. No entanto, as expectativas e pressões das partes interessadas dependem de configurações e instituições nacionais, históricas, culturais e sociais que afetarão os controles de segurança e a segurança de contas na via substancial; (ii) demonstrou a utilidade dos controles de segurança e da contabilidade na gestão das partes interessadas corporativas no setor de mineração; (iii) forneceu novos insights sobre o desempenho da sustentabilidade social corporativa em empresas de mineração e o papel e as implicações da contabilidade de sustentabilidade.

\subsubsection{RELAÇÕES DETECTADAS SOBRE DESEMPENHO}

Parece pouco provável pesquisar contabilidade de gestão da sustentabilidade e não haver relação com o tema "desempenho" nos estudos realizados, nesta pesquisa o tema foi abordado em cinco dos nove artigos dissecados. No primeiro, de Lee (2012) que também abordou controle, o tema central do artigo foi "os papéis e a utilidade do eco-controle como um meio de identificar e medir o desempenho de carbono". 
No segundo, de Lee e Wu (2014) que também abordou medição, o tema central do artigo foi "Integração de indicadores de desempenho de diferentes perspectivas e modelo de medição do sistema para melhoria do desempenho de sustentabilidade".

No terceiro, de Jasch e Lavicka (2006), o trabalho foi baseado em um projeto com o cluster de automóveis e empresas associadas selecionadas. Em seis empresas, o ambiente os custos de gestão, bem como os custos adicionais de saúde e segurança, gestão de risco e outras questões sociais foram avaliados. Menos tangíveis itens e efeitos externos são abordados. Começando com os esforços para avaliar os efeitos financeiros dos indicadores de desempenho de sustentabilidade para relatórios de sustentabilidade, o método de contabilidade de gestão ambiental foi ampliado por várias outras categorias de custos. $\mathrm{O}$ artigo descreveu estes e as experiências dos projetos-piloto, indicando que os dois principais impulsionadores de custos são os custos de aquisição da produção não relacionada ao produto e os custos relacionados à perda de dias úteis devido a licença por doença e acidentes e as horas extras pagam para compensar esses dias perdidos e que o trabalho do departamento de Saúde e Segurança Ambiental ajuda a reduzir esses custos. O esquema de avaliação de custos permite entender melhor as relações entre custos para tratamento de doenças indesejadas devido a medidas de proteção não implementadas e perda de valor de compra de material em comparação com os custos de prevenção, que consistem nos departamentos de gestão interna e consultores externos relacionados. A avaliação dos custos de gestão da sustentabilidade é de interesse das organizações, que já publicam um relatório de sustentabilidade e querem avaliar com mais precisão os efeitos financeiros de tais aspectos abordados. Também é útil para pequenas e médias empresas, que usem a avaliação como um ponto de partida para modelar seu sistema. Os principais benefícios são dados mais precisos e melhores argumentos para a avaliação de investimento ou indicadores de desempenho, bem como a melhoria da consistência dos sistemas de informação e gestão, que devem ajudá-los a melhorar seu desempenho ambiental, social e econômico.

No quarto artigo, de Silva e Guenther (2014) já mencionado na abordagem de medição, o tema central foi de "mensuração do desempenho de sustentabilidade".

E, no último artigo da abordagem sobre desempenho, de Laurinkeviciute e Stasiskiene (2011), o foco consiste nas pequenas e médias empresas (PME), as quais muitas vezes, permanecem despercebidas pois a maiorias das pesquisas costuma focar em empresas de grande porte. Além disso, as PME têm menos frequentemente conhecimento sobre seus impactos ambientais e sociais e de gestão, do que as grandes empresas. Para tornar decisões sustentáveis e melhorar o desempenho ambiental, eficácia econômica e social, uma estratégia integrada de sistema de gestão sustentável, baseado em análise é necessária, orientada para objetivos estratégicos sustentabilidades, não exigindo recursos financeiros e humanos significativos. Integração da contabilidade de gestão da sustentabilidade e metodologias compostas de índice de desenvolvimento sustentável constitui a base da tomada de decisões para as PME. A aplicação de uma estratégia integrada de sistema de gestão sustentável resultou na redução do consumo de insumos e redução dos custos de sustentabilidade.

\subsubsection{RELAÇÕES DETECTADAS SOBRE GESTÃO}

Esta abordagem teve prevalência em dois artigos e em ambas ela apareceu no sentido de gestão da sustentabilidade, no primeiro artigo, de Jasch e Lavicka (2006) o tema central do trabalho foi "a avaliação dos custos de gestão da sustentabilidade na melhoria do desempenho ambiental, social e econômico" como já citado e detalhado anteriormente. E, no primeiro artigo, de Maas et al. (2016), também já detalhado nos itens anteriores, o tema central foi "Medição e gestão de questões de sustentabilidade. 


\subsection{PROCEDIMENTOS METODOLÓGICOS E PRINCIPAIS CONCLUSÕES}

Relacionado aos procedimentos metodológicos aplicados nos artigos, estudos de caso foram comumente usados. Jasch e Lavicka (2006), Lee (2012) e Egan e Tweedie (2018), por exemplo. Normalmente, os autores realizam entrevistas e conteúdo em profundidade análise para interpretar os dados e encontrar padrões para os resultados nessas formas qualitativas de pesquisa.

A pesquisa quantitativa também foi amplamente aplicada, geralmente por meio de pesquisa por questionário, bem como uma abordagem de métodos mistos. Neste caso, uma primeira fase qualitativa e geralmente exploratória é realizada seguido de um estudo descritivo para testar um modelo. A Tabela 2 apresenta os métodos aplicados em os papéis da amostra, bem como as fontes.

Tabela 2: Principais procedimentos metodológicos utilizados na literatura

\begin{tabular}{l|l|l}
\hline Autores & Método & Fonte \\
\hline Egan e Tweedie (2018) & Estudo de caso & Accounting, Auditing \& Accountability Journal \\
\hline Gunarathne et al. (2016) & Pesquisa & Accounting Research Journal \\
\hline Jasch e Lavicka (2006) & Estudo de caso & Journal of Cleaner Production \\
\hline Laurinkeviciute e Stasiskiene (2011) & Pesquisa & Clean Techn Environ Policy \\
\hline Lee (2012) & Estudo de caso & Journal of Cleaner Production \\
\hline Lee e Wu (2014) & Métodos mistos & The British Accounting Review \\
\hline Schaltegger e Zvezdov (2015) & Pesquisa & Journal of Accounting \& Organizational Change \\
\hline Silva e Guenther (2014) & Pesquisa & $\begin{array}{l}\text { Sustainability Accounting, Management and } \\
\text { Policy Journal }\end{array}$ \\
\hline
\end{tabular}

Fonte: dados da pesquisa

De acordo com as principais conclusões os benefícios mais significativos da contabilidade de gestão da sustentabilidade são dados mais precisos e melhores argumentos, bem como, a melhoria da consistência dos sistemas de informação e gestão (JASCH e LAVICKA, 2006). Contudo, é necessário desenvolver uma abordagem integrada ligando avaliação de sustentabilidade, contabilidade de gestão, controle de gestão e relatórios (MAAS et al., 2016). Imprescindível também medir o desempenho econômico, social e ambiental, de preferência através de indicadores de desempenho que considere diferentes as diferentes perspectivas e que estejam integrados a um sistema de gestão focado no desempenho da sustentabilidade (LEE e WU, 2014). Agendas mundiais são fundamentais para a discussão e o levanto de percepções através de um diálogo colaborativo, destacando aspectos críticos que podem guiar pesquisas futuras (SILVA e GUENTHER, 2014). Os profissionais contadores podem ampliar sua contribuição para as iniciativas de sustentabilidade organizacional, se adaptando às exigências do tema, buscando mais qualificação, se envolvendo nas etapas anteriores do processo de contabilização da gestão da sustentabilidade, interagindo na manutenção entre os gestores de sustentabilidade e a alta administração e, se engajando em melhorias mais criativas (Schaltegger e Zvezdov, 2015; Egan e Tweedie, 2018).

A tabela 3, a seguir sintetiza os termos mais recorrentes entre os estudos analisados nesta pesquisa.

Tabela 3: Termos mais recorrentes na literatura

\begin{tabular}{|c|c|c|}
\hline Palavra-Chave/Variação & Recorrências & Autores \\
\hline $\begin{array}{l}\text { Sustainability management / } \\
\text { Sustainability management accounting / } \\
\text { Sustainable management / } \\
\text { Sustainable management system }\end{array}$ & 6 & $\begin{array}{l}\text { Silva e Guenther (2014); Egan e Tweedie (2018); } \\
\text { Jasch e Lavicka (2006); Laurinkeviciute e } \\
\text { Stasiskiene (2011); Schaltegger e Zvezdov } \\
\text { (2015); Laurinkeviciute e Stasiskiene (2011) }\end{array}$ \\
\hline $\begin{array}{l}\text { Sustainability accounting / } \\
\text { Sustainability accountants }\end{array}$ & 4 & $\begin{array}{l}\text { Egan e Tweedie (2018); Schaltegger e Zvezdov } \\
\text { (2015); Silva e Guenther (2014); Jasch e Lavicka }\end{array}$ \\
\hline
\end{tabular}




\begin{tabular}{l|l|l}
\cline { 3 - 3 } & & $(2006)$ \\
\hline $\begin{array}{l}\text { Carbon accounting / } \\
\text { Carbon management }\end{array}$ & 3 & Lee (2012); Lee (2012); Lee e Wu (2014) \\
\hline Automobile industry & 2 & Lee (2012); Jasch e Lavicka (2006) \\
\hline $\begin{array}{l}\text { Environmental and sustainability / } \\
\text { Environmental and sustainability } \\
\text { management accounting }\end{array}$ & 2 & Lee (2012); Lee e Wu (2014) \\
\hline $\begin{array}{l}\text { Environmental costs / } \\
\text { Environmental management accounting }\end{array}$ & 2 & Jasch e Lavicka (2006) \\
\hline $\begin{array}{l}\text { Health and safety / } \\
\text { Health and safety costs }\end{array}$ & 2 & Gunarathne et al. (2016); Jasch e Lavicka (2006) \\
\hline $\begin{array}{l}\text { Management accounting / } \\
\text { Management accounting practices }\end{array}$ & 2 & Lee (2012); Schaltegger e Zvezdov (2015) \\
\hline
\end{tabular}

Fonte: dados da pesquisa

Os termos mais recorrentes na literatura são, na maioria das vezes, sinônimos e/ou conceitos muitos semelhantes, os quais, não haveria necessidade da criação de mais de um termo, que ao invés de fortalecer o tema, o enfraquece pulverizando sua concepção para ideias sob uma mesma ótica como, por exemplo: gestão de sustentabilidade, contabilidade de gestão de sustentabilidade, gestão sustentável e sistema de gestão sustentável; ou ainda, contabilidade de carbono e gestão de carbono.

O presente estudo procurou levantar o panorama da produção acadêmica nos principais periódicos científicos sobre este tema, levantando como a contabilidade de gestão da sustentabilidade tem sido estudada na literatura e quais as principais relações detectadas, levantando os principais procedimentos metodológicos utilizados na literatura, e levantando os termos mais recorrentes utilizados na literatura. Em uma busca inicial em 34 artigos, analisados cuidadosamente, identificou-se 9 artigos que realmente estivessem dentro do foco desta análise.

Como principais achados desta pesquisa, percebeu-se que desde 2006 apenas 9 artigos foram publicados, dentre estes, abordados em quatro principais relações de Como a contabilidade de gestão da sustentabilidade tem sido estudada, a saber: (i) relações detectadas sobre os contadores, (ii) relações detectadas sobre controle e medição, (iii) relações detectadas sobre desempenho, e (iv) relações detectadas sobre gestão. Tratando-se da metodologia mais empregada nos estudos, percebeu-se os estudos de caso com maior frequência. De acordo com as principais conclusões os benefícios mais significativos da contabilidade de gestão da sustentabilidade são dados mais precisos e melhores argumentos, bem como, a melhoria da consistência dos sistemas de informação e gestão. E, por fim, como tema mais recorrente nas pesquisas percebe-se, em variadas formas a gestão da sustentabilidade.

\section{CONSIDERAÇÕES FINAIS}

Este estudo desenhou proposições para mais pesquisas empíricas sobre contabilidade de gestão da sustentabilidade. A pesquisa acadêmica tem um papel importante na melhoria da gestão da sustentabilidade e orientação para a contabilidade, fornecendo ferramentas que podem ser úteis no processo de implementação e adaptação. Identificamos como a contabilidade de gestão da sustentabilidade tem sido pesquisada na literatura. Em outras palavras, embora as empresas estejam começando a desenvolver práticas contábeis para auxiliar a gestão da sustentabilidade, a motivação ainda é muito empírica e está certamente associada ao entendimento primordial sobre sustentabilidade.

Em termos de limitações, é importante reconhecer que os resultados dependem dos critérios inicialmente usados para a seleção dos artigos, contudo existem lacunas claramente percebidas, as quais se descrevem a seguir e que, a julgar pelas mesmas não se pode afirmar 
que a contabilidade de gestão da sustentabilidade seja uma das principais ferramentas da contabilidade gerencial no auxílio à gestão da sustentabilidade, pois não existem evidências suficientes de que a contabilidade de gestão da sustentabilidade é uma das principais ferramentas da contabilidade gerencial no auxílio à gestão da sustentabilidade.

Para o universo acadêmico a pouca quantidade de publicações pode ser interpretada de duas formas, ou falta interesse em relação ao tema, ou existe um vasto campo a ser explorado. O cenário apresentado neste estudo pode auxiliar a área contábil a fazer esta reflexão.

Sobre as quatro principais relações com o tema detectadas: contadores, controle e medição, desempenho, e, gestão percebe-se o quão limitado tem sido sua exploração, uma vez que, as ciências contábeis fazem parte das ciências sociais que, por sua vez, estuda os aspectos sociais do mundo humano - a vida social de indivíduos e grupos humanos - incluindo outras áreas como antropologia, sociologia, ciência política, estudos da comunicação, marketing, administração, arqueologia, geografia humana, história, ciência da religião, contabilidade, economia, direito, psicologia social, filosofia social, e serviço social e cada uma delas pode ser investigada sob o aspectos contábil e sustentável. Além ainda, dos conhecimentos mais específicos da contabilidade como auditoria, controladoria, finanças, legislação, tributação e etc. os quais também podem ser examinados sob os mesmos aspectos.

Referente ao debate sobre a atuação e as contribuições do contador em relação à gestão da sustentabilidade, deve-se deixar um alerta, visto que este tema concentra-se na contabilidade gerencial e, se estes profissionais não se apropriarem da questão, evidenciando o domínio de habilidades e competências inter e multidisciplinares, a mesma poderá ser assumida por algum outro profissional que, até com pouco conhecimento contábil, mas com as ferramentas adequadas e a postura necessária, passa a ser um concorrente direto para ocupar uma posição que tem grandes chances de evolução, já que a necessidade de sustentabilidade organizacional é uma crescente nas empresas.

No tocante às relações da contabilidade de gestão da sustentabilidade com controle e medição fica irrefutável a necessidade de propostas de modelos que contemplem as diferentes organizações existentes, conforme ramo de atuação (indústria, comércio ou serviço); tipos de empresa (Sociedade Empresária Limitada - Ltda., Empresa Individual de Responsabilidade Limitada - Eireli, Empresa individual; Microempreendedor Individual - MEI, Sociedade Simples - SS, ou, Sociedade Anônima - SA); Porte da empresa (Microempresa - ME: fatura no máximo R \$ 360 mil por ano, Empresa de Pequeno Porte - EPP: fatura acima de R\$ 360 mil por ano até o limite de R \$ 3,6 milhões por ano, Empresas de médio porte: fatura acima de R\$ 16 até 90 milhões por ano, Empresas de médio-grande porte: fatura acima de R \$ 90 até $\mathrm{R} \$ 300$ milhões por ano, Empresas de grande porte: fatura acima de $\mathrm{R} \$ 300$ milhões por ano); número de colaboradores; tempo de atuação no mercado; entre outras questões também importantes como: perfil e grau de maturidade em relação a estratégia e governança corporativa, eficiência econômica, equilíbrio ambiental e justiça social.

A necessidade de estabelecer patamares mais elevados de desempenho estimula a reflexão acerca dos fatores que afetam o desempenho das organizações diante de um contexto marcado pela competitividade e por constantes mudanças na dinâmica mercadológica. A avaliação de desempenho consiste, assim, no julgamento decorrente do confronto dos resultados de desempenho com padrões de resultados previamente fixados, aferidos com base em indicadores de medida comuns. Já a gestão do desempenho consiste na monitorização do desempenho, nas deliberações, e na coordenação das iniciativas para conduzir o desempenho da organização ao padrão previamente estabelecido. Os desvios dos resultados constituem em oportunidades de aprendizagem organizacional. Partindo destas interpretações verifica-se, também, a necessidade de outras propostas de modelos para aferição das mais variadas formas de desempenho que as organizações podem apresentar.

E, sobre gestão nesta relação, pode-se verificar que se trata do elo entre a contabilidade 
e a sustentabilidade, sem a qual, não há como manter a sinergia entre ambos os campos de pesquisa, que por força da necessidade de atuação conjunta demonstram-se responsáveis pela perpetuação da organização.

Portanto, como sugestões para futuras pesquisas, estes itens podem ser investigados mais profundamente através de uma meta-análise, com um escopo de pesquisa mais amplo, incluindo outros bancos de dados relevantes.

Desta forma, os resultados da contabilidade de gestão da sustentabilidade teriam implicações práticas importantes para a academia, as empresas e os governos em termos de estratégicas de políticas públicas.

\section{REFERÊNCIAS}

ALMEIDA, F. O bom negócio da sustentabilidade. Rio de Janeiro: Nova Fronteira, 2002.

BARBIERI, J. C. Gestão ambiental empresarial: conceitos, modelos e instrumentos. São Paulo: Saraiva, 2007.

BARDIN, L. Análise de Conteúdo. Lisboa: Edições 70, 1977.

BURRITT, R. L.; HAHN, T.; SCHALTEGGER, S. Towards a comprehensive framework for environmental management accounting - Links between business actors and EMA tools. Australian Accounting Review, v. 12, n. 27, p. 39-50, 2002.

BURRITT, R.L.; SCHALTEGGER, S. Sustainability accounting and reporting: fad or trend? Accounting, Auditing \& Accountability. v. 23, n. 7, p. 829-846, 2010.

DIAS, S. L. F. G.; MOURA, C. Consumo Sustentável: Muito Além do Consumo "Verde". In: XXXI Encontro nacional de pós graduação em Administração (ENANPAD), 31., 2007, Rio de Janeiro. Anais eletrônicos. Rio de Janeiro, 2007, p. 1-13.

EGAN, M.; TWEEDIE, D. A "green" accountant is difficult to find: Can accountants contribute to sustainability management initiatives?. Accounting, Auditing \& Accountability v. 3, n. 6, p.1749-1773, 2018.

EPSTEIN, M. J.; BUHOVAC, A. R. Making Sustainability Work: Best Practices in Managing and Measuring Corporate Social, Environmental, and Economic Impacts. San Francisco: Berrett-Koehler, 2014.

GIL, A. C. Como elaborar projetos de pesquisa. 5. ed. São Paulo: Editora Atlas, 2010.

GUNARATHNE, N.; SAMUDRAGE, D.; WIJESINGHE, D. N.; LEE, K. Fostering social sustainability management through safety controls and accounting: A stakeholder approach in the mining sector. Accounting Research Journal. v. 29, n. 2, p. 179-197, 2016.

HÖJER, M.; AHLROTH, S.; DREBORG, K. H.; EKVALL, T.; FINNVEDEN, G.; HJELM, O.; HOCHSCHORNER, E.; NILSSON, M.; PALM, V. Scenarios in selected tools for environmental systems analysis. Journal of Cleaner Production, v. 16, n. 18, p. 1958-1970, 2008.

International Integrated Reporting Committee (IIRC). Towards integrated reporting: 
Communicating value in the 21st century. [2011]. Disponível em: $<$ http://integratedreporting.org/wp-content/uploads/2011/09/IR-Discussion-Paper-

2011_spreads.pdf $>$. Acesso em 13 out. 2018.

JASCH, C.; LAVICKA, A. Pilot project on sustainability management accounting with the Styrian automobile cluster. Journal of Cleaner Production, v. 14, n. 14, p. 1214-1227, 2006.

JITPAIBOON, T.; RAO, S. S. A meta-analysis of quality measures in manufacturing system. International Journal of Quality and Reliability Management, v. 24, n.1, p. 78-102, 2007.

LAURINKEVICIUTE, A.; STASISKIENE, Z. SMS for decision making of SMEs. Clean Techn Environ Policy, v. 13, n. 6, p. 797-807, 2011.

LEE, K. Carbon accounting for supply chain management in the automobile industry. Journal of Cleaner Production, v. 36, n. 1, p. 83-93, 2012.

LEE, K.; WU, Y. Integrating sustainability performance measurement into logistics and supply networks: A multi-methodological approach. The British Accounting Review, v.46, n. 4, p. $1-18,2014$.

LUNARDI, G, L.; FRIO, R. S.; BRUM, M. D. M. Tecnologia da Informação e Sustentabilidade: Levantamento das Principais Práticas Verdes Aplicadas à Área de Tecnologia. Gerais: Revista Interinstitucional de Psicologia, v. 4, n. 2, p. 159-172, 2011.

MAAS, K., SCHALTEGGER, S., CRUTZEN, N., Integrating corporate sustainability assessment, management accounting, control, and reporting. Journal of Cleaner Production, v. 136, n.1, p. 237-248, 2016.

MARTENS, M. L.; BRONES, F.; CARVALHO, M. M. D. Lacunas e tendências na literatura de sustentabilidade no gerenciamento de projetos: uma revisão sistemática mesclando bibliometria e análise de conteúdo. Gestão e Projetos. v. 4, n. 1, p. 165-195, 2013.

NEVES, J. L. Pesquisa qualitativa: características, usos e possibilidades. Caderno de pesquisa em administração, v. 1, n. 3., p. 1-5, 1996.

PEREIRA, F. D. S.; PEREIRA JUNIOR, E. F. Z.; BONATO, S. V.; CZARNESKI, F. R. C. Ambientes de produção enxuta sustentável: proposta de um estudo bibliométrico. In: XXIX Encontro Nacional de Cursos de Graduação em Administração (ENANGRAD), 29., 2018, São Paulo. Anais eletrônicos. São Paulo, 2018. p. 1-18.

PETTICREW, M.; ROBERTS, H. Systematic reviews in the social sciences: A practical guide. Oxford: Blackwell Publishing, 2006.

PRITCHARD, J. Statistical bibliography or bibliometrics? Journal of documentation. v. 25, n. 4, p. 348-349, 1969.

ROESCH, S. M. A. Projetos de estágio e de pesquisa em administração: guia para estágios, trabalhos de conclusão, dissertações e estudos de caso. 3. ed. São Paulo: Editora Atlas, 2010.

SAMPAIO, R. F.; MANCINI, M. C. Estudos de Revisão Sistemática: Um guia para síntese 
criteriosa da evidência científica. Brazilian Journal of Physical Therapy, v.11, n. 1, p. 83-89, 2007.

SÁNCHEZ, I. M. G.; RODRÍGUEZ, L. A.; ACEITUNO, J. V. F. The cultural system and integrated reporting. International business review. v. 22, n. 5, p. 828-838, 2013.

SCHALTEGGER, S.; ZVEZDOV, D. Gatekeepers of sustainability information: exploring the roles of accountants. Journal of Accounting \& Organizational Change, v. 11, n. 3, p. 333$361,2015$.

SEURING, S.; GOLD, S. Conducting content-analysis based literature reviews in supply chain management. Supply Chain Management: An International Journal, v. 17, n. 5, p. 544$555,2012$.

SILVA, S.; GUENTHER, E. Setting the research agenda for measuring sustainability performance - systematic application of the World Café Method. Sustainability Accounting, Management and Policy Journal, v. 9, n. 4, p. 455-469, 2018.

da SILVA, M. B. D.; BONATO, S. V.; ABRITA, N. F. M.; PEREIRA JUNIOR, E. F. Z. Análise do retorno de paletes e chapatex em empresas de bebidas. Revista latino-americana de inovação e engenharia de produção, v. 7, n. 11, p. 68-81, 2019.

TRANFIELD, D.; DENYER, D.; SMART, P. Towards a methodology for developing evidence informed management knowledge by means of systematic review. British Journal of Management, v. 14, n. 3, p. 207-222, 2003.

WORLD COMISSION ON ENVIROMENTAL AND DEVELOPMENT (WCED). Our common future. Oxford: Oxford University Press, 1987. 